\title{
The voltage-dependent anion channel-1 modulates apoptotic cell death
}

\author{
H Zaid $^{1,3}$, S Abu-Hamad ${ }^{1,3}$, A Israelson ${ }^{1}$, I Nathan ${ }^{2}$ and \\ V Shoshan-Barmatz ${ }^{*, 1,4}$ \\ ${ }^{1}$ Department of Life Sciences, Ben-Gurion University of the Negev, \\ Beer-Sheva, Israel \\ 2 Department of Clinical Biochemistry, Ben-Gurion University of the Negev, \\ Beer-Sheva, Israel \\ 3 These authors contribute equally to this work \\ ${ }^{4} \mathrm{H}$ Kreitman Chair in Bioenergetics \\ * Corresponding author: V Shoshan-Barmatz, Department of Life Sciences, \\ Ben-Gurion University of the Negev, Building 40, Beer-Sheva, Israel. \\ Tel: + 972-8-6461336; Fax: + 972-8-6472992; \\ E-mail: vardasb @ bgumail.bgu.ac.il
}

Received 08.11.04; revised 10.1.05; accepted 17.1.05; published online 01.4.05 Edited by $\mathrm{C}$ Borner

\section{Abstract}

The role of the voltage-dependent anion channel (VDAC) in cell death was investigated using the expression of native and mutated murine VDAC1 in U-937 cells and VDAC inhibitors. Glutamate 72 in VDAC1, shown previously to bind dicyclohexylcarbodiimide (DCCD), which inhibits hexokinase isoform I (HK-I) binding to mitochondria, was mutated to glutamine. Binding of HK-I to mitochondria expressing E72Q-mVDAC1, as compared to native VDAC1, was decreased by $\sim 70 \%$ and rendered insensitive to $D C C D$. HK-I and ruthenium red (RuR) reduced the VDAC1 conductance but not that of E72Q-mVDAC1. Overexpression of native or E72Q-mVDAC1 in U-937 cells induced apoptotic cell death $(80 \%)$. RuR or overexpression of HK-I prevented this apoptosis in cells expressing native but not E72Q-mVDAC1. Thus, a single amino-acid mutation in VDAC prevented HK-I- or RuR-mediated protection against apoptosis, suggesting the direct VDAC regulation of the mitochondria-mediated apoptotic pathway and that the protective effects of RuR and HK-I rely on their binding to VDAC.

Cell Death and Differentiation (2005) 12, 751-760.

doi:10.1038/sj.cdd.4401599

Published online 1 April 2005

Keywords: apoptosis; hexokinase; mitochondria; ruthenium red; VDAC

Abbreviations: ANT, adenine nucleotide translocase; G-6-P, glucose-6-phosphate; Hepes, $N$-(-hydroxyethyl])piperazine- $N$ '[2-ethanesulfonic acid]; HK, hexokinase; HK-I, hexokinase isoform I; $\beta$-OG, $n$-octyl- $\beta$-D-glucopyranoside; PLB, planar lipid bilayer; RuR, ruthenium red; STS, staurosporine; VDAC, voltagedependent anion channel; mVDAC, murine VDAC; rVDAC, rat VDAC

\section{Introduction}

Mitochondria play a crucial role in the induction of apoptosis. The release of apoptogenic intermediates such as cytochrome $c$ from the intermembrane space appears to be a central event in the initiation of the cascade that leads to programmed cell death. ${ }^{1-4}$ Mitochondrial $\mathrm{Ca}^{2+}$ overload also appears to induce both apoptotic and necrotic cell death. Mitochondria respond to an apoptotic signal by an alteration in the permeability of the mitochondrial membranes, termed permeability transition (PT), and this, in turn, may leads to apoptotic cell death. ${ }^{1-4}$ Two possible models have been proposed for this membrane permeability change. In the first model, an impairment of ATP/ADP antiport leads to hyperpolarization that promotes an osmotic matrix swelling. ${ }^{5}$ According to the second model, the PT is due to the opening of a pathway spanning both the inner and outer mitochondrial membranes. $^{2-4,6}$ The PT is thought to comprise the adenine nucleotide translocase (ANT), cyclophilin D and the voltagedependent anion channel (VDAC). ${ }^{2-4,6-10}$

The $\mathrm{Ca}^{2+}$ binding component of the permeability transition pore (PTP) has not yet been identified, although $\mathrm{Ca}^{2+}$ modulation of the ANT has been suggested. ${ }^{11}$ On the other hand, several studies have shown that VDAC is permeable to $\mathrm{Ca}^{2+}$ and possesses $\mathrm{Ca}^{2+}$ binding site(s), suggesting the involvement of these sites in the regulation of VDAC activity, as well as of PTP opening. . $^{70,12}$

Ruthenium red (RuR), a hexavalent polysaccharide stain, is known to interact specifically with $\mathrm{Ca}^{2+}$-binding proteins. $^{7,10,13-16}$ RuR has been shown to inhibit several mechanisms involved in intracellular $\mathrm{Ca}^{2+}$ regulation, including the mitochondrial uniporter and the 'rapid mode' system, ${ }^{17}$ sarcoplasmic reticulum $\mathrm{Ca}^{2+}$-ATPase activity, ${ }^{13} \mathrm{Ca}^{2+}$ release and ryanodine binding by the $\mathrm{Ca}^{2+}$ release channel/ryanodine receptor, ${ }^{14}$ the binding of $\mathrm{Ca}^{2+}$ to calsequestrin ${ }^{15}$ and to calmodulin, ${ }^{16}$ as well as to prevent brain tubulin polymerization. ${ }^{18}$ Recently, we have shown that RuR and the trivalent cation Ru360, a derivative of $\mathrm{RuR}^{19}$ can induce VDAC channel closure in a time-dependent manner, stabilizing the channel in a completely closed state. ${ }^{7,10,12}$ Both reagents also inhibit PTP opening in rat liver and retinal mitochondria. ${ }^{7,10,12}$ RuR was further shown to inhibit cytochrome $c$ release, activation of caspases-3- and curcumin-induced apoptosis in the U-937 cell line. ${ }^{20}$ Finally, RuR also inhibits both ursolic acid-induced cell death in LH- $60^{21}$ cells and microcystininduced cell death in cultured rat hepatocytes. ${ }^{22}$ However, the mechanism(s) underlying the protective action of RuR against apoptosis remains unknown.

Accumulating evidence indicates that mitochondrial-bound isoforms of hexokinase isoform I and -II (HK-I and HK-II) play a pivotal role in cancer by promoting cell growth and survival. ${ }^{23}$ Recent studies indicate that in malignant cancer, HK-II not only improves the cells energy supply but also protects against cell death. ${ }^{24}$ Moreover, HK-I has been shown to bind to a bilayer-reconstituted VDAC and to induce its 
closure, prevent mitochondrial PTP opening and prevent cytochrome $c$ release. HK-I overexpression in leukemiaderived U-937 or vascular smooth muscle cells protects against staurosporine (STS)-induced apoptotic cell death. ${ }^{25}$ HK-II has also been shown to inhibit Bax-induced cytochrome $c$ release and apoptosis in HeLa cells. ${ }^{24}$

In the present study, we have investigated the relationship between HK-I- and RuR-induced VDAC closure and protection against apoptotic cell death. A crucial role for VDAC in the regulation of apoptotic cell death that is prevented by RuR and $\mathrm{HK}-\mathrm{I}$ was demonstrated. Overexpression of VDAC1 in U-937 cells induced apoptosis that could be prevented by HK-I overexpression, RuR or Ru360 in native but not in a single amino-acid-mutated version of VDAC. These results strongly support VDAC regulation of mitochondria-mediated apoptotic pathways and show that the protective antiapoptotic effect of RuR and HK-I are mediated via their interaction with VDAC.

\section{Results}

Recently, we have shown that VDAC is highly permeable to $\mathrm{Ca}^{2+}$ and possesses $\mathrm{Ca}^{2+}$ binding sites and that VDAC channel activity is completely inhibited by both RuR and Ru360. 7,10,12 However, their binding sites in VDAC are not known. The primary amino-acid sequence of VDAC1, as deduced from its cDNA sequence, contains no features of EF-hand structures representing high-affinity $\mathrm{Ca}^{2+}$ binding sites. However, aspartate-glutamate-rich regions including residues Glu87, Asp88 form potential low-affinity $\mathrm{Ca}^{2+}$ binding sites, and, based on the proposed VDAC tertiary structure, are predicted to face the pore. ${ }^{26,27}$ To identify possible $\mathrm{Ca}^{2+}$ binding sites in VDAC and their involvement in VDAC regulation of cell life and death, recombinant murine VDAC (mVDAC) 1 or rat VDAC (rVDAC)1, modified by site-directed mutagenesis, were constructed and expressed in Saccharomyces cerevisiae (S. cerevisiae) and U-937 cells. Accordingly, recombinant native and mutated VDAC1 were expressed in the $S$. cerevisiae strain lacking VDAC1 (M222) ${ }^{28}$ from which mitochondria were isolated and tested for HKI binding, and VDACs purified from these mitochondria were tested for their bilayer-reconstituted channel activity. Finally, we examined the effect of native and mutated VDAC expression in U-937 cells on cell survival and RuR and HK-I protection against apoptotic cell death.

\section{HK-I binds to yeast mitochondria expressing native but not E72Q-mutated mVDAC1}

VDAC glutamate 72 (Glu72) was shown to interact with the carboxyl-modifying reagent dicyclohexylcarbodiimide (DCCD). ${ }^{29-31}$ Such modification was shown to inhibit HK-I binding, ${ }^{32}$ and to induce VDAC channel closure. ${ }^{30,31}$ Thus, it is expected that the HK-I binding activity of VDAC mutated at Glu72 would be reduced or eliminated. Therefore, binding of HK-I to mitochondria isolated from yeast expressing native or E72Q-mVDAC1 was compared (Figure 1a). In addition, VDACs in which Glu87, Asp88 were changed to glutamine and aspargine (E87Q, D88N) were also examined in terms of
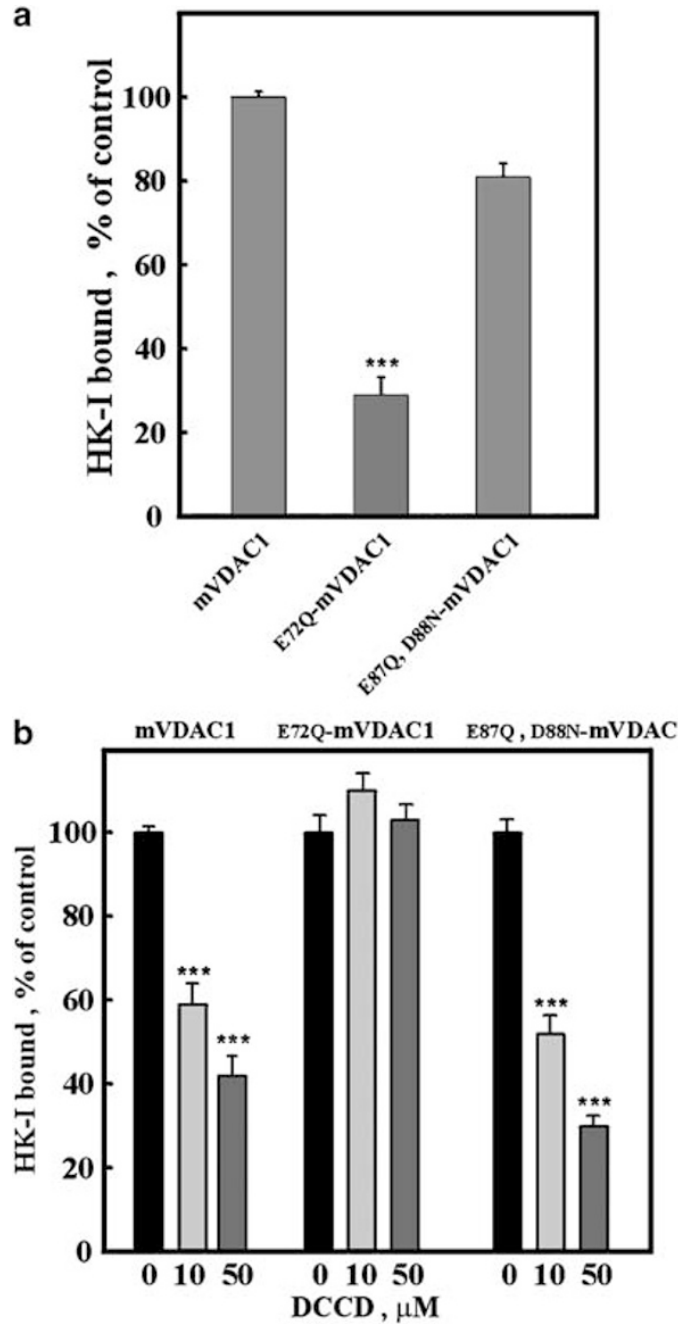

Figure $1 \mathrm{HK}-\mathrm{I}$ binding to isolated yeast mitochondria expressing native and mutated mVDAC and inhibition by DCCD. Yeast mitochondria $(2 \mathrm{mg} / \mathrm{ml})$ were incubated for $15 \mathrm{~min}$ at $25^{\circ} \mathrm{C}$ without (a) or with the indicated concentrations of DCCD (b) in a solution containing $420 \mathrm{mM}$ mannitol, $140 \mathrm{mM}$ sucrose, $10 \mathrm{mM}$ Hepes, pH 7.4 (buffer A). The treated mitochondria were centrifuged at $20000 \times g$ for $10 \mathrm{~min}$ and washed with $1 \mathrm{ml}$ buffer $A$. The mitochondria were then resuspended in $125 \mu$ incubation buffer $(0.6 \mathrm{M}$ sorbitol, $1 \mathrm{mg} / \mathrm{ml} \mathrm{BSA}, 5 \mathrm{mM}$ Mg-Hepes, pH 7.8) and $50 \mu \mathrm{l}$ of the mitochondria was incubated for $1 \mathrm{~h}$ at $4^{\circ} \mathrm{C}$ with $75 \mathrm{mU}$ HK-I. Mitochondria were centrifuged for $10 \mathrm{~min}$ at $15000 \times g$ and HK-I activity in the supernatant and the pellet was determined as described under Materials and methods. In (a), the control represents the amount of HK-I bound to the mitochondria expressing wild-type mVDAC1. HK-I bound (100\%) in the different experiments was between 30 and $35 \mathrm{mU}$ HK-I. In (b), control activity $(100 \%)$ represents the amount of HK-I bound without DCCD to mitochondria expressing native (30 and $35 \mathrm{mU})$, E72Q-mVDAC $(9-11 \mathrm{mU})$ or E87Q,D88NmVDAC $(26-29 \mathrm{mU})$. HK $(1 \mathrm{U})$ represents reduction of $1 \mu \mathrm{mol}$ of $\beta-\mathrm{NAD}^{+}$per min at $\mathrm{pH} 7.4$ at $25^{\circ} \mathrm{C}(50 \mathrm{ng} \mathrm{HK}-\mathrm{I}$ is about $1 \mathrm{mU})$. Quantitative analysis of HK-I binding was performed by ANOVA and $t$-test; $P<0.001\left(^{* \star *}\right)$ was considered statistically significant. Means \pm S.E.M. are shown $(n>5)$

HK-I binding (Figure 1a). The results show that the HK-I binding activity of E72Q-mVDAC1 was decreased by $70 \%$ in comparison to $\mathrm{HK}-\mathrm{I}$ binding to native recombinant $\mathrm{mVDAC} 1$ or E87Q,D88N-mVDAC1 (Figure 1a). Moreover, as expected, DCCD had no effect on the residual HK-I binding of the E72QmVDAC1 mutant, while it inhibited HK-I binding to mitochondria expressing native or E87Q,D88N-mVDAC1 (Figure 1b). 
HK-I interacts with native but not with E72Q-mutated purified mVDAC1 to induce channel closure

We have shown that purified $\mathrm{HK}-\mathrm{I}$ directly interacts with bilayer-reconstituted rat liver VDAC and induces its closure. ${ }^{25}$ Thus, the effect of HK-I on the channel activity of recombinant native and E72Q-mVDAC1 was considered next. The purity of the VDAC and HK-I preparations used is demonstrated in Figure 2b. As observed with native VDAC1, E72Q-mVDAC1 is stable in a long-lived, fully opened state at $-20 \mathrm{mV}$ and showed different subconductance states at $-40 \mathrm{mV}$ (Figure 2a). Similar to the results obtained with rat liverpurified VDAC ${ }^{25}$ in all experiments performed in this study, at either -20 or $-40 \mathrm{mV}$, addition of purified HK-I to recombinant native mVDAC1 induced channel closure to stable substates with a conductance reduced by $42.75 \pm 4.8 \%$ at $-20 \mathrm{mV}$ and $54 \pm 0.6 \%$ at $-40 \mathrm{mV}$ (mean \pm S.D., $n=6$ different experiments). Subsequent exposure to glucose-6-phosphate (G-6$\mathrm{P})$ reopened the HK-I-closed channel (Figure $2 \mathrm{a}$ ). In contrast, addition of HK-I to E72Q-mVDAC1 had no effect on its channel activity, nor did G-6-P addition have any effect on channel behavior (Figure 2a).

\section{RuR induces channel closure of native but not E72Q-mutated mVDAC1}

The involvement of Glu72 in the divalent cation binding site of VDAC was considered by examining the effect of the hexavalent dye RuR on VDAC channel activity. RuR is known to interact with several $\mathrm{Ca}^{2+}$-binding proteins ${ }^{13-16}$ and to inhibit $\mathrm{Ca}^{2+}$ transport in mitochondria, ${ }^{17}$ VDAC channel activity and PTP opening. ${ }^{7,10,12,17}$ Purified recombinant native and $E 72 Q-m V D A C 1$ were reconstituted into a planar lipid bilayer (PLB) as a single or multichannel and the currents produced in response to voltages stepped from a holding potential of 0 to $-20 \mathrm{mV}$ were recorded (Figure 3). As demonstrated for rat liver and retinal mitochondria, ${ }^{7,12}$ in nine of 10 different experiments performed in this study, RuR inhibited recombinant native mVDAC1 channel activity and stabilized the channel in an almost completely closed state. The level of inhibition calculated from nine different experiments was $89.2 \pm 2.8 \%$ (mean \pm S.D., $n=9$ ). In contrast, the channel activity of E72Q-mVDAC1 was not inhibited by RuR in all 10 experiments (Figure 3a) or Ru360 (data not shown), regardless of the concentrations or incubation time used. In multichannel measurements, the channel conductance of E72Q-mVDAC1 was the same in the absence or presence of RuR over a voltage range from -60 to $+60 \mathrm{mV}$ (Figure $3 \mathrm{~b}$ ). Thus, insensitivity of mutated E72Q-mVDAC1 to RuR was conferred at all voltages tested. The results indicate that Glu72 is involved in RuR interaction and in modifying VDAC channel activity.

\section{RuR protects against apoptotic cell death induced by overexpression of native but not of E72Q-mVDAC1}

RuR was shown to protect against apoptotic cell death induced by various stimuli. ${ }^{20-22}$ Such protection has been a $\quad-20 \mathrm{mV}$

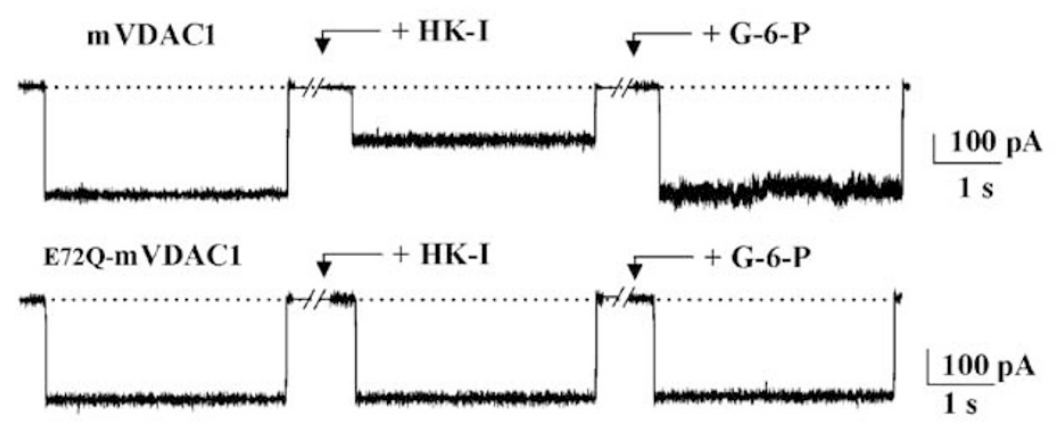

$-40 \mathrm{mV}$
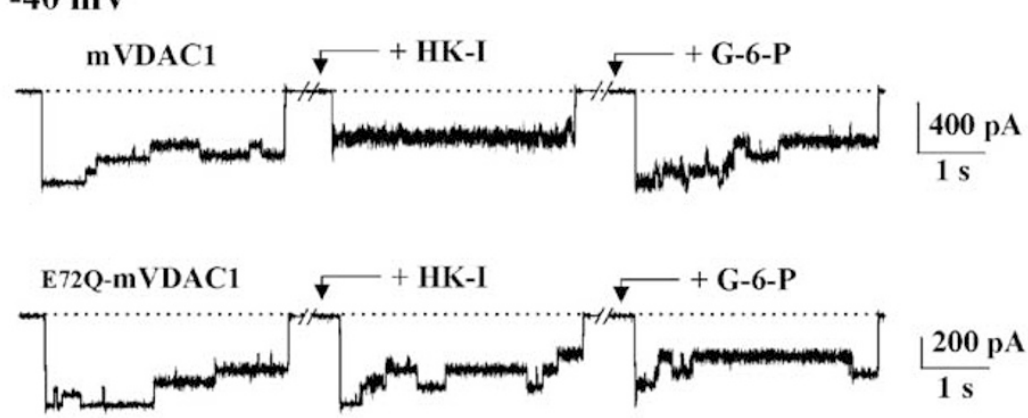

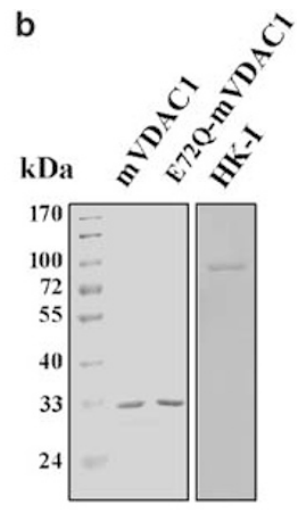

Figure 2 HK-I inhibits channel activity of native but not E72Q-mutated mVDAC1. (a) Purified recombinant native or E72Q-mutated mVDAC1 was reconstituted into a PLB in symmetrical solutions of $0.5 \mathrm{M} \mathrm{NaCl}$ as described under Materials and Methods. Currents through VDAC channel in response to a voltage step from 0 to $-20 \mathrm{mV}$ or to $-40 \mathrm{mV}$ were recorded before and $5 \mathrm{~min}$ after the addition of HK-I $(28.6 \mathrm{mU} / \mathrm{ml})$. The dashed lines indicate the zero-current level. (b) Coomassie staining of purified VDAC $(0.2 \mu \mathrm{g})$ and HK-I $(0.1 \mu \mathrm{g})$ used in these studies 

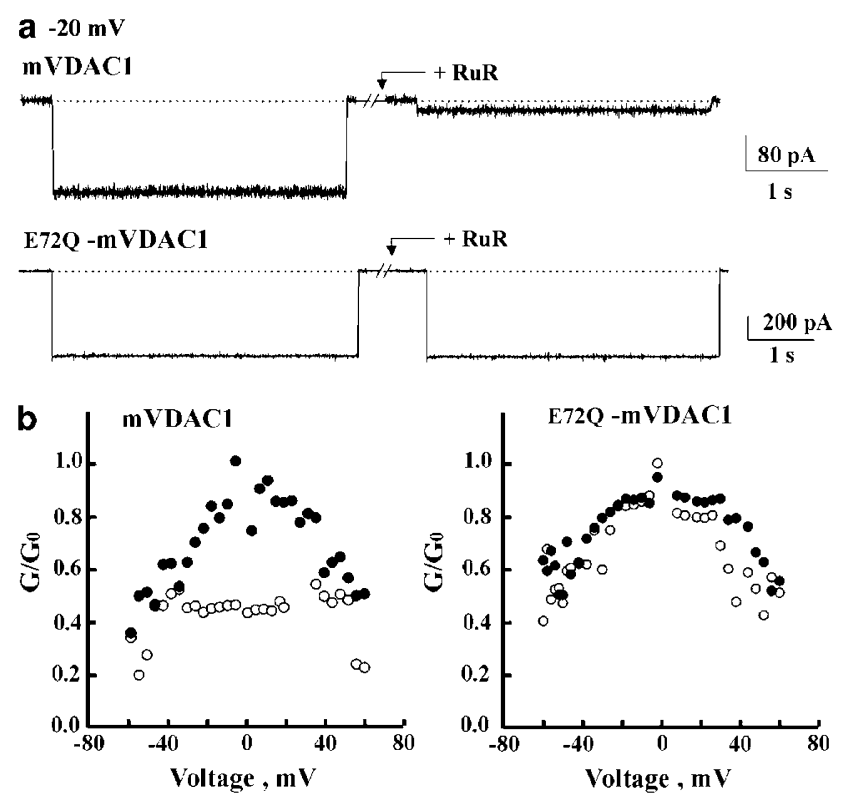

Figure 3 RuR inhibits channel activity of native but not E72Q-mutated mVDAC1. Purified native or E72Q-mVDAC1 was reconstituted into a PLB. (a) Current traces were obtained in response to voltage steps from 0 to $-20 \mathrm{mV}$. Where indicated, RuR $(50 \mu \mathrm{M})$ was added, and after $10 \mathrm{~min}$ of incubation, recordings were made. The dashed lines indicate the zero-current level. In (b), the average steady-state conductance of native or E72Q-mVDAC1 before (filled circles) and $10 \mathrm{~min}$ after the addition of $5 \mu \mathrm{M}$ Ru360 (open circles) as a function of voltage was determined by measuring the average conductance of PLB containing about 10 channels in symmetrical solutions of $1 \mathrm{M} \mathrm{NaCl}$. The conductance $(G)$ at a given voltage (from -60 to $+60 \mathrm{mV}$ ) was normalized to the conductance at $-10 \mathrm{mV}\left(G_{0}\right)$. Each point is the average of three different experiments

suggested to result from RuR binding to intracellular organelles or proteins responsible for $\mathrm{Ca}^{2+}$ buffering. However, the effect of RuR may result from its interaction with VDAC1, inducing its closure (Figure 3). Thus, to further investigate the relationship between the protective effect of RuR and its interaction with VDAC1, U-937 cells were transfected with RuR-insensitive E72Q-mVDAC1 and the effect of RuR on apoptotic cell death was addressed. First, the efficiency of cell transfection was analyzed by transforming U-937 cells to express green fluorescent protein (GFP) and VDAC1-GFP. While cells expressing GFP showed diffuse green fluorescence, cells expressing VDAC1-GFP showed mainly punctuated fluorescence confined to membranes (Figure 4c). Immunoblot analyses using anti-VDAC or antiGFP antibodies of cell extracts confirmed the expression of VDAC1-GFP (Figure 4d). Similar results were obtained with mitochondria isolated from the various cell types (data not shown). It is noteworthy that the fused VDAC1-GFP was highly protease sensitive and was readily cleaved to VDAC1 and GFP, as reflected in the appearance of GFP in the VDAC1-GFP-expressing cells (Figure 4d).

Cells transfected with native, E72Q-mutated mVDAC1 or mVDAC1-GFP, rVDAC1 or rVDAC1-GFP to induce overexpression, resulted in increased apoptotic cell death (70$75 \%$ ), as characterized by enhanced nuclear fragmentation indicative of apoptotic cell death. In contrast, control or
GFP-transfected cells showed about $14-16 \%$ nuclear fragmentation (Figure $4 a$ and $b$ and Table 1).

Preincubation of cells transfected to induce overexpression of VDAC1 or VDAC1-GFP with $1 \mu \mathrm{M}$ RuR for $18 \mathrm{~h}$ or with $5 \mu \mathrm{M}$ RuR for $5 \mathrm{~h}$ dramatically reduced their apoptotic cell death (64-72\% protection). RuR had no effect or even slightly increased apoptotic death in control or plasmid-transfected cells (Figure $4 \mathrm{~b}$ and Table 1). Strikingly, the protective effect of RuR on recombinant VDAC1 overexpression-induced cell death was not observed in cells expressing E72Q-mVDAC1 (Figure $4 b$ and Table 1). Given the RuR-insensitive behavior of E72Q-mVDAC1, as reflected by the inability of RuR to inhibit channel activity in this mutant (Figure 3), it appears that RuR protection against apoptosis is exerted through its direct interaction with VDAC. Moreover, the increase (over five-fold) in the total level of VDAC1 protein in the transfected cells was observed only upon exposure of cells expressing native mVDAC1 to RuR but not in cells expressing E72Q-mVDAC1 (Figure $4 e$ ). This indicates that the apoptotic cell death induced by VDAC1 overexpression leads to VDAC1 degradation that was prevented upon RuR binding to VDAC1 and protecting against apoptotic cell death.

To determine whether RuR could prevent not only VDAC1 overexpression-induced cell death but also cell death induced by STS, cells were preincubated for $2 \mathrm{~h}$ with either RuR or Ru360 prior to cell exposure to STS. Both RuR and Ru360 dramatically decreased STS-induced apoptotic cell death by over $60 \%$ (Table 1 ).

\section{HK-I overexpression protects against apoptotic cell death induced by overexpression of native but not of E72Q-mVDAC1}

Finally, the effect of $\mathrm{HK}-\mathrm{I}$ overexpression on apoptotic cell death of the GFP-tagged version of native and E72QmVDAC1 overexpressing cells was examined (Figure 5a). Cells were first transformed to overexpress HK-I and then transfected with native mVDAC1-GFP or E72Q-mVDAC1GFP. Apoptotic cell death induced by native mVDAC1 overexpression $(\sim 75 \%)$ was dramatically reduced in the cells overexpressing HK-I to a low level of apoptosis $(<10 \%)$, equal to the control. This protective effect of HK-I was not obtained in cells overexpressing E72Q-mVDAC1-GFP, consistent with its inability to bind HK-I (Figures 1 and 2). Immunoblot analyses indicate that mVDAC1-GFP was expressed in the transfected cells, as revealed by both antiVDAC and anti-GFP antibodies (Figure $5 b$ ). As shown, in cells transfected to stably overexpress $\mathrm{HK}-\mathrm{I}$, with and without a second transfection with mVDAC1-GFP or E72Q-mVDAC1GFP, the level of HK-I was about three- to four-fold higher than in $\mathrm{HK}-\mathrm{I}$ nontransfected cells.

\section{Discussion}

VDAC, a major mitochondrial outer membrane transporter, plays an important role in apoptosis by participating in the release of intermembrane space proteins including cytochrome $c^{3,4,6,8-10,33-35}$ Investigating the role of VDAC in cellular processes is, however, limited by a lack of tools such 
a
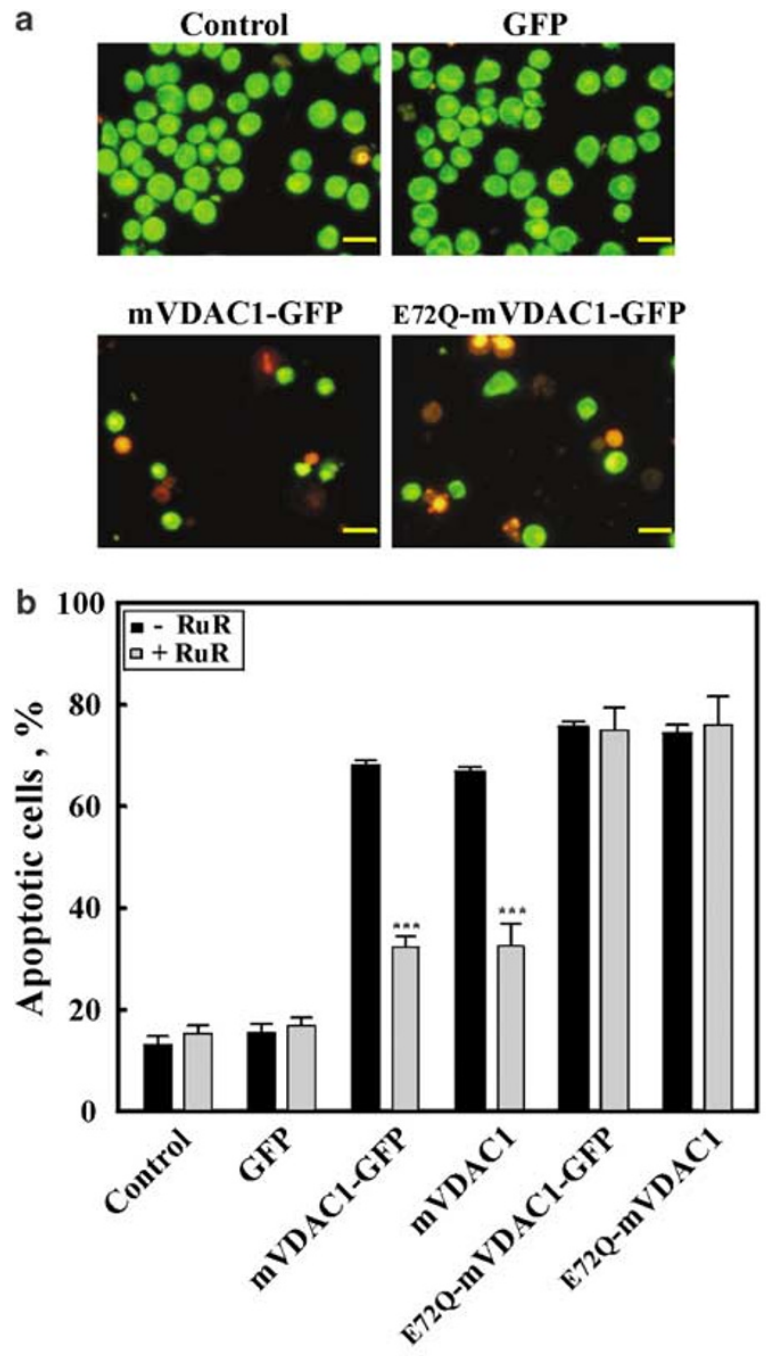

C A Control B mVDAC1-GFP

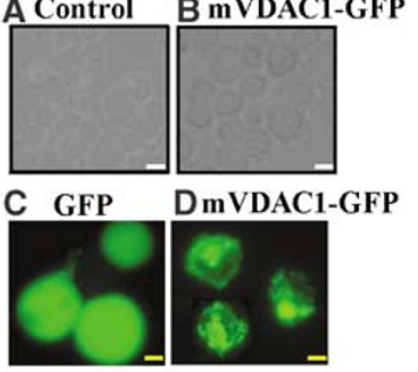

d

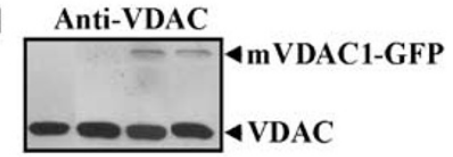

Anti-GFP

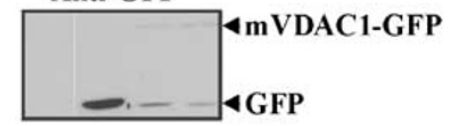

Anti-Actin

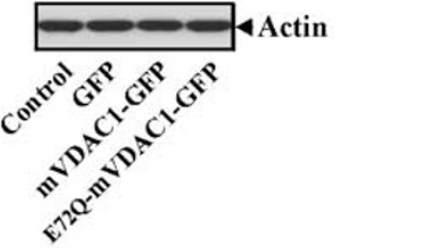

e Anti-VDAC

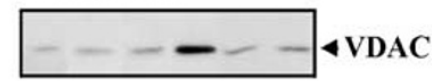

Anti-Actin

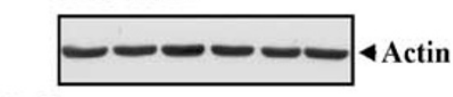

RuR - + - + - +<smiles>CCOC(C)C</smiles>

Figure 4 RuR inhibited native but not E72Q-mutated VDAC1 overexpression-induced apoptotic cell death. U-937 human monocytic cells were transfected with a GFPcontaining plasmid or a plasmid encoding native or E72Q-mutated VDAC1. (a) RuR $(1 \mu \mathrm{M})$ was added to the cells $56 \mathrm{~h}$ after transfection, and $22 \mathrm{~h}$ later, cell viability was analyzed by $\mathrm{AcOr} / \mathrm{EtBr}$ staining as described under Materials and methods (Bar $=12.5 \mu \mathrm{m})$. (b) In each independent experiment, approximately 200 cells were counted for each treatment, in which early and late apoptotic cells were also counted. Quantitative analysis of apoptosis in the different cells was performed by ANOVA and $t$-test; $\left.P<0.001{ }^{* * *}\right)$ was considered statistically significant. Data show the mean \pm S.E.M. $(n=4)$. (c) GFP or mVDAC1-GFP visualized by phase $(A+B$, bar $=1.25 \mu \mathrm{m})$ or fluorescence microscopy $(C+D$, bar $=0.30 \mu \mathrm{m}) 70 \mathrm{~h}$ after transfection. (d) Immunoblot analysis using anti-VDAC, anti-GFP or anti-actin antibodies $68 \mathrm{~h}$ after transfection. Cells were washed with PBS, resuspended in a solution containing $1 \%$ Triton X-100 and protease inhibitors, sonicated and sample aliquots (50 $\mu \mathrm{g}$ of protein) were subjected to SDS-PAGE and immunostaining. (e) Immunoblot analysis (using anti-VDAC or anti-actin antibodies) of VDAC in cells expressing VDAC1 or E72Q-mVDAC1 with and without exposure to RuR performed $78 \mathrm{~h}$ after transfection. Cell extract was obtained as in (d) and $50 \mu \mathrm{g}$ of protein was subjected to SDSPAGE

as specific, high-affinity toxins. In the present report, we have shown that RuR and Ru360, which interact with purified VDAC and inhibit its channel activity as well as the opening of the PTP, , 10,12 also inhibited apoptotic cell death induced by STS or by VDAC overexpression (Table 1, Figure 5). RuR was shown to protect against cell death induced by different stimuli including curcumin in U-937 cells, ${ }^{20}$ ursolic acid in HL-60 cells ${ }^{21}$ or microcystin in cultured rat hepatocytes. ${ }^{22}$ Since RuR interacts with $\mathrm{Ca}^{2+}$-binding proteins and inhibits their activities, ${ }^{7,10,13-18}$ the inhibition by RuR of apoptotic cell death induced by the various stimuli in different cell types suggests that apoptosis is mediated by an intracellular $\mathrm{Ca}^{2+}$ signaling pathway. The experimental data presented in this study demonstrate that RuR and Ru360 exert their protective effect on the apoptotic process by direct interaction with VDAC; thus, VDAC plays a central role in apoptotic cell death.

\section{VDAC divalent cation binding sites}

Changes in intracellular $\mathrm{Ca}^{2+}$ concentration not only stimulate a number of intercellular events but can also trigger cell death pathways including apoptosis. ${ }^{1}$ In isolated mitochondria, 


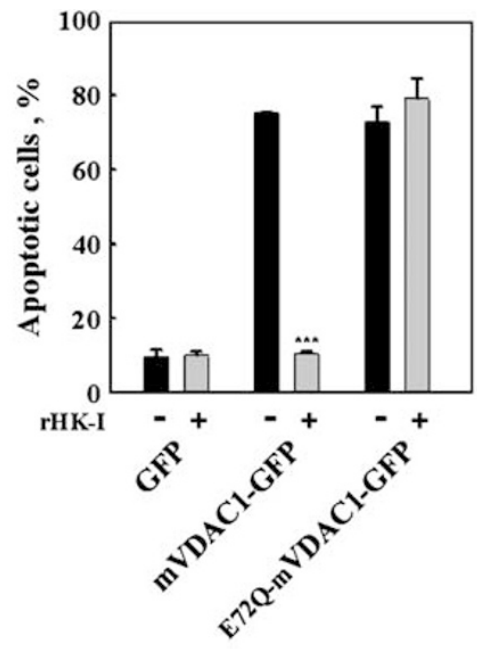

b rHK-I - - + + +

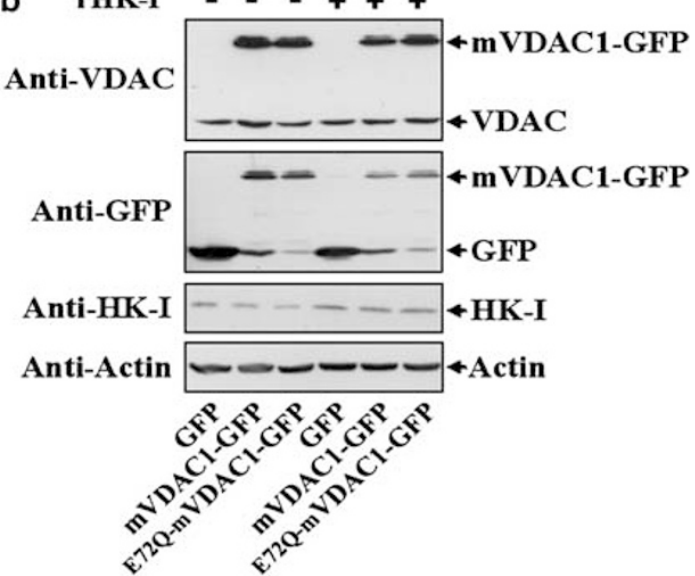

Figure 5 Overexpression of recombinant HK-I protects against apoptotic cell death induced by overexpression of native but not mutated E72Q-mVDAC1. U-937 cells were transfected with pcDNA3.1-HisA or pcDNA3.1-HisA-HK-I and grown in the presence of $400 \mu \mathrm{g} / \mathrm{ml}$ of neomycin as described under Materials and methods. The selected cells were then transfected with pEGFP, pEGFP. mVDAC1 or pEGFP-E72Q-mVDAC1 and continued to grow in the presence of neomycin $(400 \mu \mathrm{g} / \mathrm{ml})$. The viability of the double-transfected cells was analyzed after $76 \mathrm{~h}$. (a) In each independent experiment, approximately 200 cells were counted for each treatment, in which early and late apoptotic cells were also counted. Quantitative analysis of apoptosis in the different cells was performed by ANOVA and $t$-test; $\left.P<0.001{ }^{* \star *}\right)$ was considered statistically significant. Data shown are the mean \pm S.E.M. $(n=4)$. (b) Immunoblot analyses of whole cell extract (50 $\mu \mathrm{g}$ of protein) $60 \mathrm{~h}$ after transfection and using anti-VDAC, antiGFP, anti-HK-I or anti-actin antibodies

$\mathrm{Ca}^{2+}$ induces PTP opening and release of cytochrome $c .^{1-4,10}$ Currently, the mitochondrial target of $\mathrm{Ca}^{2+}$ has not yet been identified. Several lines of evidence suggest that VDAC possesses $\mathrm{Ca}^{2+}$ binding site(s) (for a review see ShoshanBarmatz and Gincel $^{10}$ ). Very little is, however, known about the $\mathrm{Ca}^{2+}$ binding site(s) of VDAC or about its function. VDAC has no EF-hand structure indicative of a high-affinity $\mathrm{Ca}^{2+}$ binding site, although potential low-affinity binding sites, composed of glutamate residues and aspartate-rich domains, are present in VDAC. Based on the proposed VDAC tertiary structure ${ }^{27}$ these residues are predicted to line the VDAC pore. In proteins, ionophores and divalent chelating reagents
Table 1 RuR protects against apoptotic cell death induced by VDAC overexpression or STS

\begin{tabular}{|c|c|c|c|}
\hline $\begin{array}{l}\text { Cells } \\
\text { transfected } \\
\text { with }\end{array}$ & $\begin{array}{l}\text { Preincubation } \\
\text { with }\end{array}$ & $\begin{array}{l}\text { Apoptotic } \\
\text { cells } \\
(\%)\end{array}$ & $\begin{array}{l}\text { Protection } \\
(\%)\end{array}$ \\
\hline \multirow{3}{*}{$\begin{array}{l}\text { None } \\
\text { pEGFP }\end{array}$} & - & $13.1 \pm 1.7$ & \\
\hline & - & $15.4 \pm 1.8$ & \\
\hline & RuR & $16.8 \pm 1.6$ & \\
\hline \multirow[t]{2}{*}{ pEGFP-rVDAC1 } & - & $42.0 \pm 3.6$ & \\
\hline & RuR & $24 \pm 2.67^{\star \star \star}$ & 72.2 \\
\hline \multirow[t]{2}{*}{ pE-rVDAC1 } & - & $44.0 \pm 3.2$ & \\
\hline & RuR & $27.1 \pm 3.2^{\star \star \star}$ & 63.6 \\
\hline \multirow[t]{2}{*}{ pEGFP-mVDAC1 } & - & $68.1 \pm 1.0$ & \\
\hline & RuR & $32.2 \pm 2.1^{\star \star \star}$ & 70.9 \\
\hline \multirow{2}{*}{ pEGFP-E72Q-mVDAC1 } & - & $75.7 \pm 1.0$ & \\
\hline & RuR & $75.2 \pm 4.5$ & 0 \\
\hline \multirow[t]{2}{*}{ None } & STS & $75.5 \pm 1.28$ & \\
\hline & $\begin{array}{l}\text { STS+RuR } \\
\text { STS+Ru360 }\end{array}$ & $\begin{array}{l}44.2 \pm 2.9^{\star \star \star} \\
54.2 \pm 3.1^{\star *}\end{array}$ & $\begin{array}{l}34.1 \\
50.2\end{array}$ \\
\hline
\end{tabular}

Control and transfected U-937 cells were incubated $2 \mathrm{~h}$ with or without RuR $(5 \mu \mathrm{M})$ or Ru360 $(5 \mu \mathrm{M})$ and then exposed to $1.25 \mu \mathrm{M}$ STS to induce apoptosis. After $7 \mathrm{~h}$, cells were stained with AcOr and $\mathrm{EtBr}$ as described in Materials and methods. In each independent experiment, approximately 200 cells were counted for each treatment, in which early and late apoptotic cells were also counted. Results are from eight experiments similar to those shown in Figure 4. Quantitative analysis of apoptosis in the different cells was performed by counting apoptotic and nonapoptotic cells. Using ANOVA and $t$-test, $P<0.01$ $\left({ }^{* *}\right)$ or $P<0.001\left(^{* * *}\right)$ was considered statistically significant. Data are means + S.E.M. $(n=8)$. The degree of protection by RuR was calculated for the VDAC transfection- or STS-induced cell death (subtracting the cell death in control)

(e.g. EGTA, EDTA), the ligation center for divalent cations binds through a carboxylate group or neutral oxygen center. DCCD, which specifically reacts with carboxyl groups, has been shown to label specifically VDAC ${ }^{29-32}$ and inhibit its channel activity. ${ }^{30,31}$ De Pinto et al. ${ }^{29}$ have identified Glu72 as the DCCD binding amino acid in VDAC. Our results show that replacement of Glu72 by glutamine eliminated RuR and Ru360 inhibition of VDAC channel activity (Figure 2) and also HK-I binding to isolated mitochondria (Figure 1). Since VDAC channel closure by RuR was prevented in the presence of $\mathrm{CaCl}_{2}$, and re-established by chelating $\mathrm{Ca}^{2+}$ with EGTA, ${ }^{7}$ and since replacement of Glu72 with glutamine resulted in a loss of RuR inhibition of VDAC channel activity, we suggest that Glu72 participates in a $\mathrm{Ca}^{2+}$ binding site(s) or is required for stabilizing VDAC in a conformation recognized by RuR, Ru360 and HK-1.

\section{A single amino-acid mutation in VDAC1 prevents HK-I- and RuR-mediated protection against cell death}

Apart from RuR (Figure 2, Table 1 and Gincel et al., 7,12 Shoshan-Barmatz and Gincel, ${ }^{10}$ Bae et al.., ${ }^{20}$ Baek et al., ${ }^{21}$ and Ding et al. ${ }^{22}$ ), overexpression of mitochondrial HK- $\left.\right|^{25}$ or $\mathrm{HK}-\mathrm{II}^{24}$ also reduces apoptosis induced by different stimuli. We have shown that HK-I and RuR directly interact with purified VDAC reconstituted into a PLB to induce channel closure. $^{7,10,12,25,31}$ Thus, it is probable that the effects of these reagents in preventing cell death are related to their interaction with VDAC. These reagents, however, are not 
specific for VDAC, and thus either the reagent or overexpression of HK-I could act via perturbation of cell physiology via other, VDAC-independent, mechanisms. Indeed, the RuRinsensitive E72Q-mutated VDAC provides an opportunity to verify the relationship between RuR interaction with VDAC and the ability of RuR to prevent apoptotic cell death. ${ }^{20-22}$ RuR-mediated protection against nuclear fragmentation in U-937 cells appeared to abrogate apoptosis by modifying VDAC function. This concept is further strongly supported by the finding that the channel activity of E72Q-mutated VDAC was not inhibited by RuR or Ru360. Expression of E72QmVDAC in U-937 cells also induced cell death that could not be prevented by RuR. Thus, although RuR can interact with different $\mathrm{Ca}^{2+}$-binding proteins in the cell, the results show that a single amino-acid mutation in VDAC can abolish RuRmediated protection against apoptosis and indicate that RuR exerts its antiapoptotic effect via a direct interaction with VDAC.

Recently, ${ }^{25}$ we have demonstrated that HK-I induces VDAC closure, suppresses the release of cytochrome $c$ and inhibits apoptotic cell death, thereby contributing to the survival advantage of tumor cells. The results presented here clearly demonstrate that a single mutation in VDAC molecule not only diminished HK-I binding to VDAC (Figures 1 and 2) but also prevented $\mathrm{HK}-\mathrm{I}$ protection against cell death induced by VDAC overexpression (Figure 5).

\section{Relationship between VDAC closed/open configuration and apoptosis}

Published results provide evidence for two apparently different hypotheses for the antiapoptotic activity of the protein $\mathrm{Bcl}-\mathrm{xL}$ : acting to promote VDAC closed conformation $^{36,37}$ or acting to stabilize VDAC open conformation, whereby its protective activity would be by restoring metabolic exchange across the outer mitochondrial membrane without inducing release of cytochrome $c .^{5,38}$

Our results show that RuR and HK-I, which reduce VDAC channel conductance, protected against cell death induced by VDAC overexpression or by STS (Azoulay-Zohar et al. ${ }^{25}$ and Figures 2-5), suggesting that decreased conductance by VDAC does not interfere with metabolite exchange between mitochondria and the cytosol, but prevents apoptotic cell death. Nevertheless, it seems that the closed/open configuration of VDAC with respect to transport of ions and metabolites is not directly applied to movement of intermembranal space proteins such as cytochrome c. A distinct VDACassociated pathway, an internal VDAC pore for movement of small molecules and a large channel formed by VDAC monomers for cytochrome $c$ release have recently been suggested. ${ }^{35}$

\section{VDAC overexpression induced apoptotic cell death}

Overexpression of mVDAC or rVDAC1 or VDAC1-GFP or E72Q-mVDAC1 in the U-937 cells resulted in cell death characterized by nuclear fragmentation. About $70-85 \%$ of the cells had died $70-78 \mathrm{~h}$ following cell transfection. Similar results were obtained upon transfection of Jurkat cells with rice or human VDAC. ${ }^{33}$ Taken together, these results indicate that the cellular expression level of VDAC may play a crucial factor in the process of mitochondria-mediated apoptosis.

The question, however, is how does enhanced VDAC expression lead to cell death? Before discussing this point, one should emphasize that an increase in the level of VDAC1 protein in the transfected cells was observed only upon exposure of cells expressing native mVDAC1 to RuR but not in cells expressing E72Q-mVDAC1 (Figure 4e). This indicates that the apoptotic cell death induced by VDAC1 overexpression leads to VDAC (both endogenous and recombinant) degradation, suggesting that VDAC becomes exposed to proteases. It is noteworthy that several proteases such as Lon and Clp are located in the mitochondria as well as in the intermembranal space. ${ }^{39}$ The finding that VDAC degradation accompanies apoptotic cell death may also suggest that the breakdown of VDAC is the primary cause of the apoptosis, although how this VDAC degradation is activated and prevented by RuR is not clear.

It is possible that a transient increase in the VDAC levels produces a significant increase in the leak of the outer mitochondrial membranes, and this, in turn, decreases cell viability. This suggestion is in line with the finding that overexpression of VDAC (by five-fold, as visualized by Western blot, Figure 4e) could only be observed upon exposure of the native- but not mutated VDAC1-transfected cells to RuR, a treatment that protects against cell death. It thus seems that it is not an increase in the total amount of VDAC but rather an increase in its functionality that is responsible for the apoptotic cell death, since RuR decreases VDAC channel conductance (Gincel et al. ${ }^{7,12}$ and ShoshanBarmatz and Gincel, ${ }^{10}$ Figure 3). Similarly, cells overexpressing HK-I, which reduces VDAC channel conductance, were resistant to VDAC overexpression-induced cell death (Figure 5). These suggest that a functional VDAC is required for triggering cell death; yet, the mechanism underlying VDAC overexpression-induced cell death is not clear.

Recently, we have shown that a dynamic VDAC oligomerization is involved in the release of cytochrome $c$ from the mitochondria and suggested that cytochrome $c$ may cross the outer mitochondrial membrane via the large flexible pore formed between individual subunits of a VDAC tetramer. ${ }^{35}$ As such, VDAC overexpression, which leads to increase in its concentration, would encourage VDAC oligomerization, and thus allow for release of proapoptotic proteins (such as cytochrome $c$ ) from the mitochondrial intermembrane space. Support for involvement of VDAC oligomerization in apoptosis is presented in recent studies on the reagent $\mathrm{As}_{2} \mathrm{O}_{3}$, where its apoptosis-inducing effect was attributed to an induction of homodimerization of VDAC molecules. ${ }^{40}$ This effect could be prevented by overexpression of the antiapoptotic protein $\mathrm{BCl}-\mathrm{xL} .{ }^{40}$ The relationship between VDAC overexpressioninduced apoptosis and VDAC oligomerization requires additional study, including direct monitoring of the oligomerization process in live cells, both in control cells and cells overexpressing VDAC.

In conclusion, our data suggest that VDAC functions as a stand-alone channel in RuR and HK-I protection against apoptotic cell death, and thus plays a key role in the regulation 
of mitochondria-mediated apoptosis. VDAC interaction with apoptosis-regulating proteins (such as HK-I) and its overexpression inducing cell death may constitute an attractive target for therapeutic intervention to suppress tumor growth.

\section{Materials and Methods}

\section{Materials}

Carboxymethyl (CM)-cellulose, n-decane, DCCD, G-6-P, glucose-6phosphate dehydrogenase (G-6-PDH), $N$-(-hydroxyethyl])piperazine- $N$ [2-ethanesulfonic acid] (Hepes), leupeptin, mannitol, PMSF, Ru360, soybean asolectin, STS, sucrose and Tris were purchased from Sigma (St. Louis, MO, USA). Cibacron blue-agarose was purchased from Amersham Biosciences (Uppsala, Sweden). n-Octyl- $\beta$-D-glucopyranoside ( $\beta$-OG) was obtained from Bachem AG (Bubendorf, Switzerland). Lauryl(dimethyl)-amineoxide (LDAO) and RuR (97\% pure) were obtained from Fluka (Buchs, Switzerland). Hydroxyapatite (Bio-Gel HTP) was purchased from Bio-Rad Laboratories (Hercules, CA, USA) and Celite from the British Drug Houses (UK). Monoclonal anti-VDAC antibodies raised against the $\mathrm{N}$-terminal region of $31 \mathrm{HL}$ human porin (clone no. $173 / 045$, Cat. No. 529538-B) came from Calbiochem-Novobiochem (Nottingham, UK). Monoclonal antibodies against actin and against the GFPs were obtained from Santa Cruz Biotechnology (Santa Cruz, CA, USA). Polyclonal antibodies against HK were kindly provided by Dr. John $E$ Wilson (University of Michigan, Ann Arbor, MI, USA). Horseradish peroxidase (HRP)-conjugated goat anti-rabbit antibodies were obtained from Zymed (San Francisco, CA, USA). HRP-conjugated anti-mouse antibodies were obtained from Promega (Madison, WI, USA).

\section{Yeast strains, media and cultivation conditions}

The expression of native and mutated mVDAC genes was conducted in the S. cerevisiae por1- mutant strain M22-2 (MATa, lys2 his4 trp1 ade2 $\left.u_{\mathrm{ura3}}\right)^{28}$ under the control of the yeast porin1 (YVDAC1) promoter in a lowcopy number plasmid (pSEYC58). S. cerevisiae M22-2 and its evolved mutant were cultured at $30^{\circ} \mathrm{C}$ in selective minimal medium containing ammonium sulfate, yeast nitrogen base, the required amino acids and $2 \%$ glucose or in rich medium containing 1\% yeast extract, $2 \%$ peptone (YP) with $2 \%$ lactate (YPL) or glucose (YPD) at pH 5.5. Yeast M22-2 expressing either native or mutated mVDAC1 cultured in medium containing glucose showed a similar and high rate of growth (not shown). For HK-I binding, mitochondria were isolated from yeast cultured in lactate containing rich medium (to induce mitochondria biogenesis). Cultures were stored in aliquots supplemented with $25 \%$ glycerol at $-80^{\circ} \mathrm{C}$ and were revived in YPD medium at $30^{\circ} \mathrm{C}$

\section{Plasmids and site-directed mutagenesis}

mVDAC1 cDNAs (obtained from WJ Craigen, University of Houston, Texas, USA) were subcloned into plasmid pEGFP-N1 (Clontech) for construction of VDAC1-GFP or VDAC1 expressing mammalian vector with a neomycin-resistant gene serving as marker. Rat muscle VDAC1 cDNA was cloned and sequenced (V Shoshan-Barmatz and R Ashley, unpublished). Site-directed mutagenesis of mVDAC1 was carried out in vitro by overlapping PCR amplification. Recombinant plasmid pSEYC58, carrying wild-type mVDAC1 gene, served as the template for amplification of the mutant VDAC genes. The mutated mVDAC1 genes were constructed using the T7 and T3 universal primers, together with the following primer: 5'-GACGTTTACACAGAAGTGGAAC-3' (forward) and
5'-GTTCCACTTCTGTGTAAACGTC-3' (reverse) to introduce the E72Q mutation and 5'-TCACTGTGCAGAACCAGCTTGC-3' (forward) and 5'AAGCTGGTTCTGCACAGTGATC $\bar{C} 3^{\prime}$ (reverse) to introduce the E87Q $\mathrm{D} 88 \mathrm{~N}$ mutations. All constructs were confirmed by sequencing.

\section{Mitochondrial preparation}

Mitochondria were isolated from yeast ${ }^{41}$ or U-937 cells ${ }^{42}$ using published procedures.

\section{Protein determination}

Mitochondrial protein content was determined by the Biuret method, ${ }^{43}$ while hexokinase concentration was determined according to Bradford, ${ }^{44}$ using ovalbumin as the standard.

\section{Rat brain HK-I purification, activity and binding to mitochondria}

Rat brain HK-I was purified as described previously. ${ }^{25} \mathrm{HK}-\mathrm{I}$ activity was assayed by coupling NADH formation to the production of G-6-P by HK-I and its subsequent oxidation by G-6-PDH in a reaction mixture containing $4 \mathrm{mM}$ Mg-Hepes, $1 \mathrm{mM} \mathrm{K-EDTA}, 0.6 \mathrm{mM} \mathrm{NAD}^{+}, 10 \mathrm{mM}$ glucose, $1 \mathrm{mM}$ ATP, $1 \mathrm{mg} / \mathrm{ml}$ BSA, $20 \mathrm{mM}$ K-Hepes, $\mathrm{pH}$ 7.8. HK-I binding to mitochondria isolated from the different yeast strains $(2 \mathrm{mg} / \mathrm{ml})$ was carried out by a $1 \mathrm{~h}$ incubation on ice of the mitochondria with HK-I $(0-2 \mathrm{U} / \mathrm{ml})$ in $0.1 \mathrm{ml}$ of a solution containing $420 \mathrm{mM}$ mannitol, $140 \mathrm{mM}$ sucrose, $1 \mathrm{mg} / \mathrm{ml} \mathrm{BSA}$, $5 \mathrm{mM} \mathrm{Mg-Hepes,} \mathrm{pH}$ 7.8. Soluble and mitochondria-bound HK-I-containing fractions were separated and analyzed for HK-I activity. ${ }^{25} \mathrm{HK}-\mathrm{I}$ binding was defined as the percent of activity present in the bound fraction relative to the total activities found in the bound and free fractions combined.

\section{Purification of VDAC}

Native and mutated mVDAC1 were extracted with LDAO from mitochondria isolated from yeast expressing various VDACs, and purified by chromatography on hydroxyapatite followed by CM-cellulose where LDAO was replaced by $\beta$-OG. ${ }^{10}$

\section{VDAC channel recording and analysis}

Reconstitution of purified VDAC into a PLB, multichannel current recording and data analyses were carried out as described previously. ${ }^{7}$ Briefly, PLB was prepared from soybean asolactin dissolved in $n$-decane $(50 \mathrm{mg} / \mathrm{ml})$ in a chamber containing $10 \mathrm{mM} \mathrm{Hepes} / \mathrm{KOH}(\mathrm{pH} 7.4)$ and 0.5 or $1 \mathrm{M} \mathrm{NaCl}$ (cis/trans). Only PLBs with a resistance greater than $100 \mathrm{G} \Omega$ were used. Purified VDAC (about $1 \mathrm{ng}$ ) was added to the chamber defined as the cis side. After one or a few channels were inserted into the PLB, excess protein was removed by perfusion of the cis chamber with 20 volumes of a solution (with the same composition as before perfusion), in order to prevent further protein incorporation. Currents were recorded under voltage-clamp mode using a Bilayer Clamp amplifier (Warner Instrument, Hamden, CT, USA). The currents were measured with respect to the trans side of the membrane (ground). The currents were low-pass filtered at $1 \mathrm{kHz}$, using a Bessal filter (Frequency Devices, Haverhill, MA, USA) and digitized on-line using a Digidata 1200 interface board and PCLAMP 6 software (Axon Instruments, Union City, CA, USA). Sigma Plot 2000 scientific software was used for data analyses. Experiments were performed at $23-25^{\circ} \mathrm{C}$. 


\section{Gel electrophoresis and immunoblot analyses}

SDS-PAGE was performed according to Laemmli. ${ }^{45}$ Gels were stained with Coomassie Brilliant blue or electroblotted onto nitrocellulose membranes ${ }^{46}$ and immunostained using monoclonal anti-VDAC, antiGFP, anti-actin or polyclonal anti-HK-I antibodies followed by incubation with HRP-conjugated anti-mouse IgG for anti-VDAC, anti-GFP or antiactin antibodies, and HRP-conjugated goat anti-rabbit lgG for anti-HK-I as secondary antibodies. Antibody binding was detected by chemiluminescence using a kit obtained from Santa Cruz Biotechnology (Santa Cruz, CA, USA).

\section{Tissue culture}

The U-937 human monocytic cell line was grown under an atmosphere of $95 \%$ air and $5 \% \mathrm{CO}_{2}$ in RPMI 1640 supplemented with $10 \%$ fetal calf serum (FCS), $1 \mathrm{mM} \mathrm{L-glutamine,} 100 \mathrm{U} / \mathrm{ml}$ penicillin and $100 \mu \mathrm{g} / \mathrm{ml}$ streptomycin.

\section{Cell transfection}

Logarithmically growing U-937 cells were resuspended in RPMI 1640 supplemented with $10 \%$ FCS, $100 \mathrm{U} / \mathrm{ml}$ penicillin and $100 \mu \mathrm{g} / \mathrm{ml}$ streptomycin at a concentration of $2.5 \times 10^{7}$ cells $/ \mathrm{ml}$. Cells were transfected to express rmVDAC1 or mVDAC1 using the vectors pEGFP, pEGFP-VDAC1, pEGFP-E72Q-mVDAC1, pE-mVDAC1 or pE-E72QmVDAC1. Transfection was performed by electroporation with a single pulse from a Bio-Rad micropulser II with a capacitance extender unit $(200 \mathrm{~V}, 950 \mu \mathrm{F})$. Cells were incubated on ice for $10 \mathrm{~min}$ before and after transfection, and then resuspended in $20 \mathrm{ml}$ of RPMl 1640 supplemented with $10 \%$ FCS, $1 \mathrm{mM}$ L-glutamine, $100 \mathrm{U} / \mathrm{ml}$ penicillin and $100 \mu \mathrm{g} / \mathrm{ml}$ streptomycin. Transfection efficiencies were $68-72 \%$, as assessed by GFP expression. GFP-positive cells were viewed under a microscope using a blue filter.

\section{Double transfection}

U-937 cells were transfected with pcDNA3.1-HisA or pcDNA3.1-HisA-HK-I (neomycin resistance; provided by Dr. JE Wilson, University of Michigan) by electroporation, and grown in complete RPMI 1640 with neomycin $(400 \mu \mathrm{g} / \mathrm{ml})$. After 12 days, the selected cells were transfected with either pEGFP, pEGFP-mVDAC1 or pEGFP-E72Q-mVDAC1 and were grown in the presence of neomycin $(400 \mu \mathrm{g} / \mathrm{ml})$. The viability of double-transfected cells was analyzed during $48-76 \mathrm{~h}$.

\section{Treatment of cells}

Cells were plated at a density of $5.4 \times 10^{4} \mathrm{cell} / \mathrm{s} / \mathrm{cm}^{2}$ in 24-well plates, washed once with PBS and placed in serum-free medium. Cells were then incubated with or without RuR or Ru360, under conditions indicated in the legend to figures and table. Cells were also treated with $1.25 \mu \mathrm{M}$ STS to induce apoptosis and analyzed for viability 3,5 and $7 \mathrm{~h}$ after STS addition.

\section{Acridine Orange (AcOr)/ethidium bromide (EtBr) staining of cells}

To determine cell viability, cells were subjected to staining with $100 \mu \mathrm{g} / \mathrm{ml}$ AcOr and $100 \mu \mathrm{g} / \mathrm{ml} \mathrm{EtBr}$ in PBS. ${ }^{47}$ Cells were centrifuged at $1500 \times \mathrm{g}$ for $5 \mathrm{~min}$ at room temperature and resuspended in $25 \mu \mathrm{l}$ of complete medium, to which $2 \mu \mathrm{l}$ of $\mathrm{AcOr} / \mathrm{EtBr}$ solution was added. The cells were then visualized by fluorescence microscopy (Olympus IX51) and images were recorded on an Olympus DP70 camera, using an SWB filter.

\section{Acknowledgements}

This research was supported by a grant from the Israel Science Foundation, administrated by The Israel Academy of Science and Humanities. We gratefully acknowledge the assistance of Dr. Sara Sivan.

\section{References}

1. Berridge MJ, Bootman MD and Lipp P (1998) Calcium - a life and death signal. Nature 395: 645-648

2. Kroemer G, Zamzami N and Susin SA (1997) Mitochondrial control of apoptosis. Immunol. Today 18: 44-51

3. Crompton M, Virji S, Doyle V, Johnson N and Ward JM (1999) The mitochondrial permeability transition pore. Biochem. Soc. Symp. 66: 167-179

4. Halestrap AP, Doran E, Gillespie JP and O'Toole A (2000) Mitochondria and cell death. Biochem. Soc. Trans. 28: 170-177

5. Vander Heiden MG, Chandel NS, Schumacker PT and Thompson CB (1999) $\mathrm{Bcl}-\mathrm{xL}$ prevents cell death following growth factor withdrawal by facilitating mitochondrial ATP/ADP exchange. Mol. Cell 3: 159-167

6. Zoratti M and Szabo I (1995) The mitochondrial permeability transition. Biochim. Biophys. Acta 1241: 139-176

7. Gincel D, Zaid H and Shoshan-Barmatz V (2001) Calcium binding and translocation by the voltage-dependent anion channel: a possible regulatory mechanism in mitochondrial function. Biochem. J. 358: 147-155

8. Bernardi P (1999) Mitochondrial transport of cations: channels, exchangers, and permeability transition. Physiol. Rev. 79: 1127-1155

9. Cesura AM, Pinard E, Schubenel R, Goetschy V, Friedlein A, Langen H, Polcic $P$, Forte MA, Bernardi P and Kemp JA (2003) The voltage-dependent anion channel is the target for a new class of inhibitors of the mitochondrial permeability transition pore. J. Biol. Chem. 278: 49812-49818

10. Shoshan-Barmatz V and Gincel D (2003) The voltage-dependent anion channel: characterization, modulation, and role in mitochondrial function in cell life and death. Cell Biochem. Biophys. 39: 279-292

11. Brustovetsky $N$ and Klingenberg $M$ (1996) Mitochondrial ADP/ATP carrier can be reversibly converted into a large channel by $\mathrm{Ca}^{2+}$. Biochemistry 35 : 8483-8488

12. Gincel D, Vardi N and Shoshan-Barmatz V (2002) Retinal voltage-dependent anion channel: characterization and cellular localization. Invest. Ophthalmol. Vis. Sci. 43: 2097-2104

13. Corbalan-Garcia S, Teruel JA and Gomez-Fernandez JC (1992) Characterization of ruthenium red-binding sites of the $\mathrm{Ca}^{2+}$-ATPase from sarcoplasmic reticulum and their interaction with $\mathrm{Ca}^{2+}$-binding sites. Biochem. J. 287: $767-774$

14. Chen SR and MacLennan DH (1994) Identification of calmodulin, $\mathrm{Ca}^{2+}$ and ruthenium red-binding domains in the $\mathrm{Ca}^{2+}$ release channel (ryanodine receptor) of rabbit skeletal muscle sarcoplasmic reticulum. J. Biol. Chem. 269: 22698-22704

15. Charuk JH, Pirraglia CA and Reithmeier RA (1990) Interaction of ruthenium red with $\mathrm{Ca}^{2+}$-binding proteins. Anal. Biochem. 188: 123-131

16. Sasaki T, Naka M, Nakamura F and Tanaka T (1992) Ruthenium red inhibits the binding of calcium to calmodulin required for enzyme activation. J. Biol. Chem. 267: 21518-21523

17. Gunter TE, Buntinas L, Sparagna GC and Gunter KK (1998) The $\mathrm{Ca}^{2+}$ transport mechanisms of mitochondria and $\mathrm{Ca}^{2+}$ uptake from physiological-type $\mathrm{Ca}^{2+}$ transients. Biochim. Biophys. Acta 1366: 5-15

18. Deinum J, Wallin M, Kanje M and Lagercrantz C (1981) The effect of ruthenium red on the assembly and disassembly of microtubules and on rapid axonal transport. Biochim. Biophys. Acta 675: 209-213

19. Ying WL, Emerson J, Clarke MJ and Sanadi DR (1991) Inhibition of mitochondrial calcium ion transport by an oxo-bridged dinuclear ruthenium ammine complex. Biochemistry 30: 4949-4952 
20. Bae JH, Park JW and Kwon TK (2003) Ruthenium red, inhibitor of mitochondrial $\mathrm{Ca}^{2+}$ uniporter, inhibits curcumin-induced apoptosis via the prevention of intracellular $\mathrm{Ca}^{2+}$ depletion and cytochrome $c$ release. Biochem. Biophys. Res. Commun. 303: 1073-1079

21. Baek JH, Lee YS, Kang CM, Kim JA, Kwon KS, Son HC and Kim KW (1997) Intracellular $\mathrm{Ca}^{2+}$ release mediates ursolic acid-induced apoptosis in human leukemic HL-60 cells. Int. J. Cancer 73: 725-728

22. Ding WX, Shen HM and Ong CN (2001) Pivotal role of mitochondrial $\mathrm{Ca}^{2+}$ in microcystin-induced mitochondrial permeability transition in rat hepatocytes. Biochem. Biophys. Res. Commun. 285: 1155-1161

23. Bryson JM, Coy PE, Gottlob K, Hay N and Robey RB (2002) Increased hexokinase activity, of either ectopic or endogenous origin, protects renal epithelial cells against acute oxidant-induced cell death. J. Biol. Chem. 277: 11392-11400

24. Pastorino JG, Shulga N and Hoek JB (2002) Mitochondrial binding of hexokinase II inhibits Bax-induced cytochrome $c$ release and apoptosis. J. Biol. Chem. 277: 7610-7618

25. Azoulay-Zohar H, Israelson A, Abu-Hamad S and Shoshan-Barmatz V (2004) In self-defence: hexokinase promotes voltage-dependent anion channel closure and prevents mitochondria-mediated apoptotic cell death. Biochem. J. 377: 347-355

26. Reymann S, Florke H, Heiden M, Jakob C, Stadtmuller U, Steinacker P, Lalk VE, Pardowitz I and Thinnes FP (1995) Further evidence for multitopological localization of mammalian porin (VDAC) in the plasmalemma forming part of a chloride channel complex affected in cystic fibrosis and encephalomyopathy. Biochem. Mol. Med. 54: 75-87

27. Song $J$ and Colombini $M$ (1996) Indications of a common folding pattern for VDAC channels from all sources. J. Bioenerg. Biomembr. 28: 153-161

28. Blachly-Dyson E, Song J, Wolfgang WJ, Colombini $M$ and Forte $M$ (1997) Multicopy suppressors of phenotypes resulting from the absence of yeast VDAC encode a VDAC-like protein. Mol. Cell. Biol. 17: 5727-5738

29. De Pinto V, al Jamal JA and Palmieri F (1993) Location of the dicyclohexylcarbodiimide-reactive glutamate residue in the bovine heart mitochondrial porin. J. Biol. Chem. 268: 12977-12982

30. Shafir I, Feng W and Shoshan-Barmatz V (1998) Dicyclohexylcarbodiimide interaction with the voltage-dependent anion channel from sarcoplasmic reticulum. Eur. J. Biochem. 253: 627-636

31. Shoshan-Barmatz V, Hadad N, Feng W, Shafir I, Orr I, Varsanyi M and Heilmeyer LM (1996) VDAC/porin is present in sarcoplasmic reticulum from skeletal muscle. FEBS Lett. 386: 205-210

32. Nakashima RA, Mangan PS, Colombini M and Pedersen PL (1986) Hexokinase receptor complex in hepatoma mitochondria: evidence from $N$, $N^{\prime}$-dicyclohexylcarbodiimide-labeling studies for the involvement of the poreforming protein VDAC. Biochemistry 25: 1015-1021
33. Godbole A, Varghese J, Sarin A and Mathew MK (2003) VDAC is a conserved element of death pathways in plant and animal systems. Biochim. Biophys. Acta 1642: 87-96

34. Tsujimoto $Y$ and Shimizu S (2002) The voltage-dependent anion channel: an essential player in apoptosis. Biochimie 84: 187-193

35. Zalk R, Israelson A, Garty E, Azoulay-Zohar H and Shoshan-Barmatz V (2005) Oligomeric states of the voltage-dependent anion channel and cytochrome $c$ release from mitochondria. Biochem. J. 386: 73-83

36. Shimizu S, Narita M and Tsujimoto Y (1999) Bcl-2 family proteins regulate the release of apoptogenic cytochrome $c$ by the mitochondrial channel VDAC. Nature 399: 483-487

37. Shimizu S, Konishi A, Kodama T and Tsujimoto $Y(2000) B H 4$ domain of antiapoptotic Bcl-2 family members closes voltage-dependent anion channel and inhibits apoptotic mitochondrial changes and cell death. Proc. Natl. Acad. Sci. USA 97: 3100-3105

38. Vander Heiden MG, Li XX, Gottleib E, Hill RB, Thompson CB and Colombini M (2001) Bcl-XL promotes the open configuration of the voltage-dependent anion channel and metabolite passage through the outer mitochondrial membrane. J. Biol. Chem. 276: 19414-19419

39. Bota AD and Davies KJA (2001) Protein degradation in mitochondria: implications for oxidative stress, aging and disease: a novel etiological classification of mitochondrial proteolytic disorders. Mitochondrion 1: 33-49

40. Zheng Y, Shi $Y$, Tian C, Jiang C, Jin H, Chen J, Almasan A, Tang $\mathrm{H}$ and Chen $\mathrm{Q}$ (2004) Essential role of the voltage-dependent anion channel (VDAC) in mitochondrial permeability transition pore opening and cytochrome $c$ release induced by arsenic trioxide. Oncogene 23: 1239-1247

41. Lee AC, Xu X, Blachly-Dyson E, Forte M and Colombini M (1998) The role of yeast VDAC genes on the permeability of the mitochondrial outer membrane. J. Membr. Biol. 161: 173-181

42. Bourgeron T, Chretien D, Rotig A, Munnich A and Rustin P (1992) Isolation and characterization of mitochondria from human $B$ lymphoblastoid cell lines. Biochem. Biophys. Res. Commun. 186: 16-23

43. Gornall AG, Bardawill CJ and David MM (1949) Determination of serum proteins by means of the Biuret reaction. J. Biol. Chem. 177: 751-766

44. Bradford MM (1976) A rapid and sensitive method for the quantitation of microgram quantities of protein utilizing the principle of protein-dye binding. Anal. Biochem. 72: 248-254

45. Laemmli UK (1970) Cleavage of structural proteins during the assembly of the head of bacteriophage T4. Nature 227: 680-685

46. Towbin H, Staehelin T and Gordon J (1979) Electrophoretic transfer of proteins from polyacrylamide gels to nitrocellulose sheets: procedure and some applications. Proc. Natl. Acad. Sci. USA 76: 4350-4354

47. McGahon AJ, Martin SJ, Bissonnette RP, Mahboubi A, Shi Y, Mogil RJ, Nishioka WK and Green DR (1995) The end of the (cell) line: methods for the study of apoptosis in vitro. Methods Cell Biol. 46: 153-185 